\title{
Magnetic and backbone exponents of the percolation and Ising models in three dimensions
}

\author{
Youjin Deng ${ }^{1, *}$ and Henk W. J. Blöte ${ }^{1,2}$ \\ ${ }^{1}$ Faculty of Applied Sciences, Delft University of Technology, P. O. Box 5046, 2600 GA Delft, The Netherlands \\ ${ }^{2}$ Lorentz Institute, Leiden University, P. O. Box 9506, 2300 RA Leiden, The Netherlands
}

(Received 17 May 2004; published 14 October 2004)

\begin{abstract}
We investigate random-cluster representations of the $q=1$ - and 2-state Potts models in three dimensions, i.e., the bond-percolation and the Ising model, respectively. Using a recently developed sampling technique, we determine the probabilities $C_{1}(r)$ and $C_{2}(r)$ that a pair of lattice sites at a distance $r$ are connected by at least one and two mutually independent paths, respectively. The scaling behavior of $C_{1}$ and $C_{2}$ at criticality is governed by the magnetic and the backbone scaling dimension $X_{h}$ and $X_{b}$, respectively. From a finite-size analysis of the numerical data, we determine $X_{h}=0.4768$ (7) and $X_{b}=1.125(3)$ for the percolation and $X_{h}$ $=0.5178(7)$ and $X_{b}=0.829(4)$ for the Ising model.
\end{abstract}

DOI: 10.1103/PhysRevE.70.046106

PACS number(s): 05.50.+q, 64.60.Cn, 64.60.Fr, 75.10.Hk

\section{INTRODUCTION}

The Ising model and the percolation problem have for many years been of great interest to physicists and mathematicians. While the Ising model presents a very simple theoretical description [1] for thermodynamic phase transitions in magnetic systems, the percolation provides a fascinating illustration [2] of many important concepts of critical phenomena in terms of geometric properties. Both models can be exactly mapped onto the random-cluster model [3], in which thermodynamic singularities of the Ising model can also be represented in terms of percolation clusters. For an introduction, we start with the Hamiltonian of the $q$-state Potts model [4] on the square lattice

$$
\mathcal{H} / k_{B} T=-K \sum_{\langle i, j\rangle} \delta_{\sigma_{i}, \sigma_{j}} \quad(\sigma=1,2, \ldots, q),
$$

where the sum is over nearest-neighbor (NN) spins and $K$ is the coupling strength. The random-cluster model is obtained as follows. Between each pair of NN sites, a bond is placed with the probability $p=1-\exp (-K)$, so that the whole lattice is decomposed into connected clusters, i.e., the well-known Kasteleyn-Fortuin (KF) clusters [3]. The statistical weight of each bond-variable configuration is given by the partition function of the random-cluster model

$$
Z_{r c}(q ; K)=\sum_{b} v^{n_{b}} q^{n_{c}} \quad\left(v=e^{K}-1\right) .
$$

Here, the sum is over all bond-variable configurations, and $n_{b}$ and $n_{c}$ are the total numbers of bonds and KF clusters, respectively. It can be shown [3-5] that the partition sum of the Potts model (1) is equivalent to $Z_{r c}$ in Eq. (2). The Ising and percolation models are the special cases with $q=2$ and $q \rightarrow 1$, respectively. Near the critical point $K_{c}(q)$, the scaling properties of KF clusters in Eq. (2) are governed by the thermal and magnetic scaling fields.

\footnotetext{
*Present address: Laboratory for Material Science, Delft University of Technology, Rotterdamseweg 137, 2628 AL Delft, The Netherlands.
}

In addition to the thermal and magnetic scaling dimensions $X_{t}$ and $X_{h}$, there are still a number of critical exponents that characterize structural properties of critical KF clusters in Eq. (2) and do not have a thermodynamic analog. Among them there are fractal dimensions [6] of "backbones" and of "red" bonds. Here, we shall briefly review definitions of these quantities in the language of the bond-percolation model in two dimensions. Consider an $L \times L$ square lattice with periodic and fixed boundary conditions in the $x$ and $y$ directions, respectively, i.e., the so-called bus-bar geometry. If a potential difference is applied to the "bars" $y=0$ and $y$ $=L$ and the aforementioned bond variables are regarded as conducting units, the backbone then consists of those bonds that would carry a current and the so-called "Wheatstone bridges." The current vanishes on these "bridges" accidentally because of zero potential difference between their ends. If a bond carries all the current and thus becomes "hot" after some time, this bond is then named a red bond [6]. At criticality, the mass (the total number of bonds) of red bonds and that of the backbone scale as $N_{r} \propto L^{d-X_{r}}$ and $N_{b} \propto L^{d-X_{b}}$, respectively. Here, $d=2$ is the lattice dimension, and $X_{r}$ and $X_{b}$ are termed the red-bond and the backbone scaling dimension, respectively. Apparently, all the red bonds are on the backbone, and the backbone is only a part of the KF cluster which connects the bars $y=0$ and $y=L$. This yields the inequality $X_{h} \leqslant X_{b} \leqslant X_{r}$. For a KF cluster with one or more red bonds, it will, if any of the red bonds is eliminated, split into disconnected subclusters. Therefore, the magnitudes of $X_{b}$ and $X_{r}$ reflect the "compactness" of a critical KF cluster.

For the general $q$-state Potts model in two dimensions, the nature of thermodynamic singularities is now well established. For instance, the thermal and magnetic dimensions $X_{t}$ and $X_{h}$ can be obtained exactly from the Coulomb gas theory [7,8]. A large amount of information is also available for geometric exponents. For instance, the red-bond dimension $X_{r}$ has already been identified with the exponent [9-11] that governs the renormalization flow of the bond probability $p$. As a result, exact values of $X_{r}$ can be included in the prediction of the conformal field theory $[12,13]$. However, except for some special cases, e.g., the uniform spanning tree ( $q$ $\rightarrow 0$ ) [4], the backbone dimension $X_{b}$ has not been exactly 
obtained. Numerous theoretical attempts have been carried out. It was conjectured $[14,15]$ that $X_{b}=7 / 16$ for the percolation model, which, however, has been falsified by numerical tests. More recently, $X_{b}$ is related [16] to the solution of a partial differential equation, which appears to be intractable even numerically.

In parallel with these theoretical attempts, several numerical determinations of $X_{b}$ have been reported, including Monte Carlo [17-20] and transfer-matrix [21] analyses. One of the Monte Carlo methods uses the so-called burning algorithm and evaluates the mass of the backbone $N_{b}$ in the aforementioned bus-bar geometry. From the scaling behavior of $N_{b}$, Grassberger [18] determined that $X_{b}=0.3569$ (8) for the two-dimensional percolation model. For the $q=2$ - and 3 -state Potts models in two dimensions, it was estimated [19] that $X_{b}=0.25(1)$ and $0.25(2)$, respectively.

Recently, another Monte Carlo approach was proposed [20] which makes use of a different formulation of the backbone problem. It has been shown [22-24] that the backbone problem can be related to so-called $k$-connected clusters, where $k \geqslant 1$ is an integer. A cluster is $k$ connected if, by eliminating any $k-1$ sites, no separation into disconnected subclusters is possible. Thus, any two sites in such a cluster are connected via at least $k$ independent paths without any bond in common. Near criticality, the scaling behavior of the mass of these $k$-connected clusters is dominated by a family of critical exponents $X_{k}$. It is obvious that the aforementioned KF clusters defined in Eq. (2) are just one type of oneconnected cluster, so that $X_{1}$ is just the magnetic dimension $X_{h}$. Moreover, it can be shown [24] that $X_{2}$ is equal to the backbone dimension $X_{b}$. In the bus-bar geometry, this can be understood as follows. According to the definition of the backbone mentioned earlier, a site on the backbone must be connected to both bars by at least two mutually independent paths, where "independence" means that these paths do not have any bond in common. For an infinite system, if one replaces the bars simply by a point, the backbone is then related to the problem concerning two mutually independent paths. On the basis of the relation $X_{2}=X_{b}$, an efficient sampling technique was developed [20] to investigate the probability $C_{2}(r)$ that a pair of sites at a distance $r$ are connected via at least two independent paths. The quantity $C_{2}$ was referred [20] to as the "backbone correlation function," and moreover the backbone dimension $X_{b}$ has been determined for several critical Potts models in two dimensions, including $q=2-\sqrt{3}, 1,2,3$, and 4. Moreover, it was argued [20] that, for the whole tricritical branch of the two-dimensional Potts model, the backbone dimension $X_{b}$ is equal to the magnetic dimension $X_{h}$. Numerical confirmation of this equivalence was also provided [20]. This is due to the fact that, in this case, the red-bond scaling field is irrelevant $\left(X_{r}>2\right)$.

In comparison with the two-dimensional case, exact results are scarce for critical behavior in three dimensions. Therefore, investigations have to depend on approximations such as $\epsilon$ and series expansions, and Monte Carlo techniques. Extensive studies have been carried out and significant results have been achieved [2,25-34]. For instance, the percolation threshold of the bond percolation on the simple-cubic lattice was determined [26] as $p_{c}=0.2488216(5)$, and the backbone dimension was reported [35] as $X_{b}$ $=1.145(15)$. From quantities such as the mean cluster size, the thermal and magnetic scaling dimensions were estimated [2,25-27] as $X_{t}=1.859(3)$ and $X_{h}=0.477(3)$, respectively. By means of conformal invariance and simulations of the anisotropic limit of the bond-percolation model defined on a spherocylinder, it was recently reported that $X_{h}=0.479$ (1) [36]. For the Ising model, there is also some consensus [28-34] that the values of $X_{t}$ and $X_{h}$ are 0.413 and 0.518, respectively, with differences only in the last decimal place. However, it seems that so far little attention has been given to the geometric exponents of the Ising model, such as $X_{b}$ and $X_{r}$.

In this paper, we present, using the technique developed in Ref. [20], a numerical study of the backbone problem for the percolation and the Ising model in three dimensions. The models and the Monte Carlo procedures are briefly reviewed in Sec. II. Section III presents the finite-size analysis, and thus the results for $X_{h}$ and $X_{b}$ for both models. A brief discussion is given in Sec. IV.

\section{MODELS AND METHODS}

\section{A. Dilute Ising model}

We start with the Hamiltonian of a dilute Ising model on the simple-cubic lattice

$$
\mathcal{H} / k_{B} T=-K \sum_{\langle i, j\rangle} s_{i} s_{j}+D \sum_{k} s_{k}^{2} \quad(s=0, \pm 1) .
$$

The spins assume the values \pm 1 and 0 . Those in state $s=0$ are referred to as vacancies. The abundance of vacancies is controlled by the chemical potential $D$, and nonzero couplings $K$ occur only between NN Ising spins. For $D \rightarrow-\infty$, the vacancies are excluded, and the model reduces to the "pure" Ising model, i.e., the spin- $\frac{1}{2}$ model [1]. This model has been investigated extensively, and the critical point was determined [34] as $K_{c}=0.22165455(3)$. Along the critical line $K_{c}(D)$, the amplitude of the irrelevant scaling field with the exponent $y_{i}=-0.821(5)$ varies as a function of $D$.

It was reported [28,34] that this amplitude is very small near $D=\ln 2$, and thus the present paper investigates the dilute Ising model (3) with $D=\ln 2$. At this point, the critical

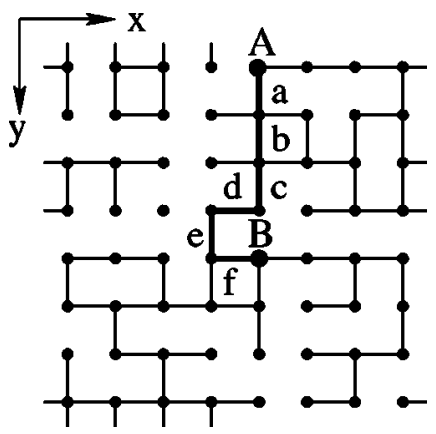

FIG. 1. An illustration of the KF cluster and the path $\mathrm{P}$ for the case of $w=2$. The system size is $L=8$, and the occupied bonds crossing the periodic boundaries are shown at both sides as half the length of the whole bond. 


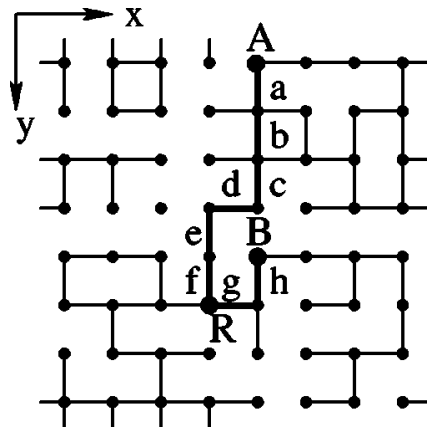

FIG. 2. An illustration of the KF cluster and the path $\mathbb{P}$ for the case of $w=1$. The system size is $L=8$. The bonds $g$ and $h$ are red bonds.

coupling and the corresponding vacancy density were determined $[28,34]$ as $K_{c}=0.39342225(5)$ and $\rho_{v c}$ $=0.400694(1)$, respectively. During the simulations, we fixed the global density of vacancies at the critical value $\rho_{v c}$, while they are still allowed to move freely over the lattice according to the Boltzmann distribution. This means that an external constraint is imposed. For such a constrained system, it is known [37] that the thermal exponent $y_{t}$ $=1.5868(3)$ [34] is renormalized to $y_{t}^{\prime}=X_{t}=1.4132(3)$. Thus, the constrained specific heat has only a finite cusp at criticality $[37,38]$ instead of being divergent. Large-scale simulations of the model (3) under the constraint are now possible because of the so-called geometric cluster method [39]. This algorithm is developed on the basis of geometric symmetries such as the spatial-inversion symmetry, and has been explained in Ref. [39]. The geometric cluster simulations of these constrained systems suffer little from critical slowing down. This may be related to the fact that the constrained critical specific heat takes a finite value.

\section{B. Sampling procedure}

The sampling procedure for the backbone correlation function $C_{2}(r)$ has already been described in detail in Ref. [20]. For completeness, it will be briefly recalled as follows.

For simplicity, we consider a bond percolation on the $L$ $\times L$ square lattice with periodic boundary conditions. Then, a site $A$ is randomly chosen, and the site at a distance $L / 2$ from $A$ in the $x$ direction is denoted as $B$. According to the aforementioned definition of $C_{2}$, the task is then to determine how many independent paths exist between $A$ and $B$. We introduce a variable $w=0,1$, and 2 , representing the cases that there is no path, precisely one path, and at least two mutually independent paths. For this purpose, one grows a KF cluster from site $A$. This is done similarly as in the standard Wolff [40] steps for the Ising model. However, since we are interested in the backbone problem, one has to store the bond variables. For system size $L=8$ and the bond probability $p$ $=1 / 2$, Figs. 1 and 2 illustrate two typical and similar KF clusters, where the occupied bonds are represented by solid lines.

After completeness of the $\mathrm{KF}$ cluster, if site $B$ is not in the cluster, then $A$ and $B$ are not connected. Thus, one has $w$ $=0$, and the current sampling procedure is completed; otherwise, one continues as follows.

A path $\mathrm{P}$ is formed from $A$ to $B$, and this is done [20] by a "smart ant" which walks from $A$ via the occupied bonds that are already stored in computer memory. The ant can always arrive at its destination $B$, since it is in the KF cluster. We illustrate the paths $P$ in Figs. 1 and 2 as thick lines.

If only one independent path can be formed, at least one red bond occurs between $A$ and $B$ and, moreover, it must be on the path $\mathrm{P}$. Therefore, the remaining task is to check whether there is any red bond on $\mathrm{P}$. This can be done as follows. Temporarily eliminate all the bonds on the path $\mathbb{P}$, and let the ant restart its journey. In the case of Fig. 1, the ant can still arrive at its destination $B$ via the periodic boundaries. Thus, no red bond exists on $\mathbb{P}$, and one has $w=2$. In Fig. 2, after the elimination of path $\mathbb{P}$, the ant cannot reach $B$ but it can still arrive at site $R$. This means that the bonds $a, b, \ldots$, and $f$ are not red bonds, and thus they can be restored. With the restoration of these bonds, the ant can continue its journey, but it cannot go beyond site $R$, because bond $g$ is a red bond. In this case, the variable $w=1$.

It was shown [20] that the computer time cost by this sampling procedure is in the same order of that by a standard Wolff step [40]. Moreover, for the Ising model, it occupies only a relatively small fraction of the total time since additional simulations are needed. With the variable $w$, one can then define

$$
C_{1}=\left\langle\delta_{w, 1}+\delta_{w, 2}\right\rangle \text { and } C_{2}=\left\langle\delta_{w, 2}\right\rangle,
$$

where \langle\rangle means the statistical average. The quantity $C_{1}$ is the probability that site $A$ is connected to $B$, and represents the magnetic correlation function. The quantity $C_{2}$ is just the aforementioned backbone correlation function.

TABLE I. The data for $C_{1}$ and $C_{2} \times 10$ for the critical bond-percolation model in three dimensions. The numbers in parentheses are the statistical errors in the last decimal place.

\begin{tabular}{ccccccccc}
\hline \hline & 6 & 8 & 10 & 12 & 14 & 16 & 18 & 20 \\
\hline$C_{1}$ & $0.20747(3)$ & $0.15496(2)$ & $0.12411(2)$ & $0.10378(2)$ & $0.08926(1)$ & $0.07839(1)$ & $0.06996(1)$ & $0.06321(1)$ \\
$C_{2}$ & $0.13899(6)$ & $0.06889(4)$ & $0.04049(3)$ & $0.02641(2)$ & $0.01846(2)$ & $0.01359(2)$ & $0.01039(2)$ & $0.00815(1)$ \\
& 24 & 28 & 32 & 36 & 40 & 48 & 60 & 80 \\
\hline$C_{1}$ & $0.05301(1)$ & $0.045719(9)$ & $0.040200(9)$ & $0.035905(9)$ & $0.032465(9)$ & $0.027260(8)$ & $0.022031(8)$ & $0.016723(8)$ \\
$C_{2}$ & $0.00537(1)$ & $0.003801(8)$ & $0.002803(8)$ & $0.002149(7)$ & $0.001694(7)$ & $0.001117(6)$ & $0.000676(5)$ & $0.000354(5)$ \\
\hline \hline
\end{tabular}




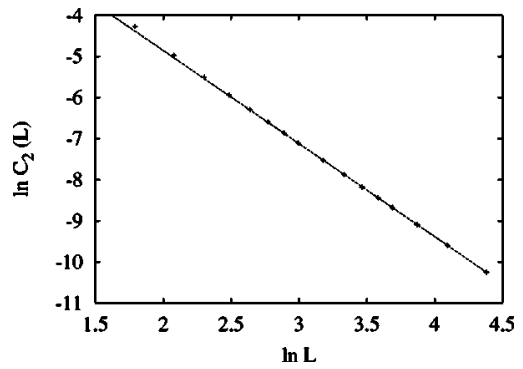

FIG. 3. The backbone correlation function $C_{2}$ for the bondpercolation model, shown as $\ln C_{2}(L)$ vs $\ln L$.

\section{RESULTS}

\section{A. Bond-percolation model}

The bond-percolation model was investigated on $L \times L$ $\times L$ simple-cubic lattices with periodic boundary conditions, and the bond probability $p$ was set at the critical value [26] $p_{c}=0.2488216(5)$. The magnetic and backbone correlation functions $C_{1}(L / 2)$ and $C_{2}(L / 2)$ were sampled for three pairs of points in the $x, y$, and $z$ directions, respectively, and the average values were calculated. The system sizes were chosen as 16 values in the range $6 \leqslant L \leqslant 80$, and a number of $2.1 \times 10^{7}$ samples was taken for each system size. The numerical data for $C_{2}(L / 2)$ are listed in Table I. In comparison with the magnetic correlations $C_{1}(L / 2)$, the backbone correlations $C_{2}(L / 2)$ are relatively small, which indicates that critical KF clusters are rather "ramified." According to the least-squares criterion, we fitted the data for $C_{1}(L / 2)$ and $C_{2}(L / 2)$ by

$$
C_{1}(L / 2)=L^{-2 X_{h}}\left(a_{0}+a_{1} L^{y_{i}}+a_{2} L^{-2}+a_{3} L^{-3}\right)
$$

and

$$
C_{2}(L / 2)=L^{-2 X_{b}}\left(b_{0}+b_{1} L^{y_{i}}+b_{2} L^{-2}+b_{3} L^{-3}+b_{4} L^{y_{i b}}\right),
$$

where $a_{i}$ and $b_{i}$ are unknown parameters. The correction terms with exponent $y_{i}$ arise from the irrelevant scaling field, of which the exponent $y_{i}$ has been determined [26] as $y_{i}=$ $-1.14(15)$. For the backbone correlation $C_{2}$, new finite-size corrections could appear, and thus we include the term with the exponent $y_{i b}$ in Eq. (6). Figure 3 shows the data for $C_{2}$ on a logarithmic scale, i.e., $\ln C_{2}(L / 2)$ versus $\ln L$. The approxi-

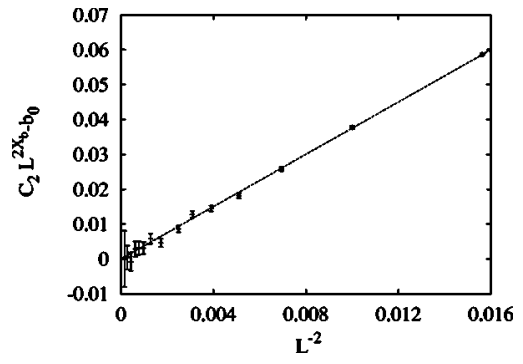

FIG. 4. Illustration of finite-size corrections in the backbone correlation $C_{2}$ for the bond-percolation model. This is shown as $C_{2} L^{2 X_{b}}-b_{0}$ vs $L^{y_{i b}}$, where the values of $X_{b}, b_{0}$, and $y_{i b}$ are taken from the numerical fit: $X_{b}=1.125(3), b_{0}=0.688(13)$, and $y_{i b}$ $=-2.0(5)$.

mate linearity indicates that corrections to scaling are not very significant.

If the exponent $y_{i}$ is left free during the fit for the magnetic correlation $C_{1}$, we have $X_{h}=0.4769(6)$ and $y_{i}$ $=-1.5(3)$, where the quoted error margins are two standard deviations, as obtained from the statistical analysis. The estimation of $y_{i}$ is consistent with $y_{i}=-1.14(15)$ [26]. The fit with $y_{i}$ fixed at -1.14 yields that $X_{h}=0.4768(4)$. Taking into account the uncertainties of the percolation threshold $p_{c}$, we conclude that $X_{h}=0.4768(8)$ is a reasonable estimation, which is in good agreement with existing results $X_{h}$ $=0.477$ (3) [25-27]. In the fit for the backbone correlation $C_{2}$, it seems unnecessary to include all correction terms described by Eq. (6), because not all of them can be well determined, and moreover results do not depend on whether they are present or not. After excluding the terms with $a_{1}$ and $a_{2}$, we obtain $X_{b}=1.125(3)$ and $y_{i b}=-2.0(5)$, which improves significantly over the existing estimation $X_{b}=1.145(15)$ [35]. The fit to the data for $C_{2}$ is illustrated by Fig. 4 .

We mention that, in Eqs. (5) and (6), the analytic corrections with exponents -2 and -3 are included just because the data fits indicate the existence of corrections decaying faster than the term with $y_{i}$. In these equations, the corrections with exponents $m y_{i}-n$ can occur, in principle, where $m>0$ and $n>0$ are integers. Therefore, in the analysis of the numerical data, we made several tries by using different combinations of correction exponents. We find that the results for $X_{h}$ and $X_{b}$ do not sensitively depend on these combinations.

TABLE II. The data for $C_{1}, C_{2} \times 10$, and $C_{22} \times 100$ for the dilute Ising model in three dimensions. The numbers in parentheses are the statistical errors in the last decimal place.

\begin{tabular}{cccccccc}
\hline \hline & 6 & 8 & 10 & 12 & 14 & 16 & 18 \\
\hline$C_{1}$ & $0.15598(3)$ & $0.11571(3)$ & $0.09214(2)$ & $0.07656(2)$ & $0.06550(2)$ & $0.05722(2)$ & $0.05081(2)$ \\
$C_{2}$ & $0.31726(9)$ & $0.18909(9)$ & $0.12878(8)$ & $0.09479(8)$ & $0.07339(7)$ & $0.05881(6)$ & $0.04850(6)$ \\
$C_{22}$ & $0.4668(2)$ & $0.2151(2)$ & $0.1211(1)$ & $0.07649(9)$ & $0.05212(8)$ & $0.03758(7)$ & $0.02818(6)$ \\
& 20 & 24 & 28 & 32 & 40 & 48 & $0.01873(1)$ \\
\hline$C_{1}$ & $0.04565(1)$ & $0.03794(1)$ & $0.03243(1)$ & $0.02832(1)$ & $0.02257(1)$ & $0.01395(1)$ \\
$C_{2}$ & $0.04081(5)$ & $0.03030(5)$ & $0.02359(4)$ & $0.01893(3)$ & $0.01318(2)$ & $0.00979(2)$ & $0.00613(2)$ \\
$C_{22}$ & $0.02170(5)$ & $0.01394(5)$ & $0.00956(4)$ & $0.00689(3)$ & $0.00400(2)$ & $0.00258(1)$ & $0.00125(1)$ \\
\hline \hline
\end{tabular}




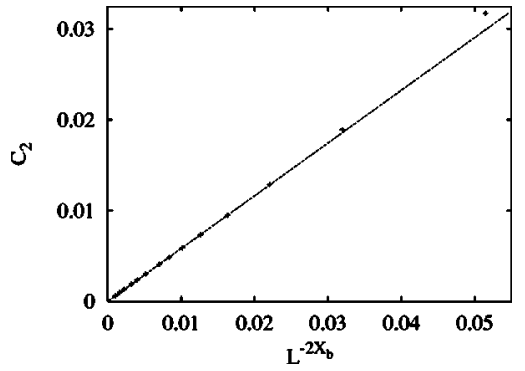

FIG. 5. The backbone correlation function $C_{2}$ for the Ising model, shown as $\ln C_{2}$ vs $L^{-2 X_{b}}$, where $X_{b}=0.829(5)$ is the backbone scaling dimension.

\section{B. Dilute Ising model}

The dilute Ising model (3) on the simple-cubic lattice was also investigated with periodic boundary conditions. Several Monte Carlo cluster steps were performed between subsequent samples. The simulations took place at the critical point $[28,34] \rho_{v c}=0.400694(1)$ and $K_{c}=0.39342225(5)$. As mentioned earlier, the total number of vacancies is fixed at $V_{c}=L^{2} \rho_{v c}$, and thus a combination of the Wolff [40] and the geometric [39] cluster steps was used. For finite systems $L$, however, $V_{c}$ is not an integer. Thus, the actual simulations were performed at two numbers $\left[V_{c}\right]$ and $\left[V_{c}\right]+1$, where the brackets [ ] denote the integer part. Numerical data at criticality were obtained by linear interpolation between $\left[V_{c}\right]$ and $\left[V_{c}\right]+1$. System sizes were taken as 14 values in the range $6 \leqslant L \leqslant 64$. Apart from the quantities $C_{1}$ and $C_{2}$, we also sampled the probability $C_{22}$ that the randomly chosen site $A$ is simultaneously connected to $B$ and $D$ by at least two mutually independent paths, where $B$ and $D$ are two points at a distance $L / 2$ in the $x$ and $y$ directions, respectively. The data for $C_{1}, C_{2}$, and $C_{22}$ are listed in Table II. The scaling behavior of $C_{22}$ is described by

$$
C_{22}(L / 2)=L^{-3 X_{b}}\left(x_{0}+x_{1} L^{y_{i}}+x_{2} L^{-2}+x_{3} L^{-3}+x_{4} L^{y_{i b}}\right) .
$$

The irrelevant exponent $y_{i}$ was set at $-0.821(5)$ [34], and the data for $C_{1}$ were fitted by Eq. (5). We obtain $X_{h}=0.5178(7)$, in agreement with the earlier estimation $X_{h}=0.5184(1)$ [34], which was obtained from the magnetic susceptibility. For the backbone problem, we simultaneously fitted Eqs. (6) and (7) by the data for $C_{2}$ and $C_{22}$, respectively, such that the backbone dimension $X_{b}$ appears only once. As in the case of the bond-percolation model, we found no evidence that new correction terms with $y_{i b}$ exist. We obtain $X_{b}=0.829(4)$, where the quoted error is again twice the standard deviation. The data for $C_{2}$ are shown in Fig. 5 versus $L^{-2 X_{b}}$, where $X_{b}$ $=0.829$ was taken from the fit.

\section{DISCUSSION}

In conclusion, we have numerically determined the backbone dimension $X_{b}$ for the bond-percolation and Ising models in three dimensions. As the thermal and magnetic dimensions $X_{t}$ and $X_{h}$, geometric critical exponents are also universal, and thus our results for $X_{b}$ should also apply to other systems within the percolation and Ising universality class in three dimensions.

In addition to the backbone and red-bond dimensions $X_{b}$ and $X_{r}$, there are other exponents characterizing geometric properties of critical systems, e.g., the fractal dimension $X_{\min }$ of "chemical" paths [17]. In percolation theory, these exponents have received significant attention, and they are considered to be of some physical relevance. For instance, the chemical-path dimension $X_{\min }$ is the analog in percolation of the dynamic scaling exponent of critical phenomena [17]. However, further explorations of the geometric exponents seem appropriate for other critical systems in three dimensions.

\section{ACKNOWLEDGMENTS}

We are indebted to Dr. J. R. Heringa and X. F. Qian for useful discussions. We are particularly indebted to Professor B. Nienhuis for his valuable contributions during the development of the sampling procedure. This research was supported by the Dutch FOM foundation ("Stichting voor Fundamenteel Onderzoek der Materie"), which is financially supported by the NWO ("Nederlandse Organisatie voor Wetenschappelijk Onderzoek").
[1] L. Onsager, Phys. Rev. 65, 117 (1944).

[2] D. Stauffer and A. Aharony, Introduction to Percolation Theory (Taylor \& Francis, Philadelphia, 1994), and references therein.

[3] P. W. Kasteleyn and C. M. Fortuin, J. Phys. Soc. Jpn. 46 (Suppl.), 11 (1969); C. M. Fortuin and P. W. Kasteleyn, Physica (Amsterdam) 57, 536 (1972).

[4] For a review, see, e.g., F. Y. Wu, Rev. Mod. Phys. 54, 235 (1982).

[5] A. Coniglio and W. Klein, J. Phys. A 12, 2775 (1980).

[6] H. J. Herrmann and H. E. Stanley, Phys. Rev. Lett. 53, 1121 (1984); H. J Herrmann, D. C. Hong, and H. E. Stanley, J. Phys. A 17, L261 (1984).

[7] B. Nienhuis, J. Phys. A 15, 199 (1982).
[8] See, e.g., B. Nienhuis, in Phase Transitions and Critical Phenomena, edited by C. Domb and J. L. Lebowitz (Academic, London, 1987), Vol. 11, p. 1, and references therein.

[9] A. Coniglio, Phys. Rev. Lett. 62, 3054 (1989).

[10] H. W. J. Blöte, Y. M. M. Knops, and B. Nienhuis, Phys. Rev. Lett. 68, 3440 (1992).

[11] Y. Deng, H. W. J. Blöte, and B. Nienhuis, Phys. Rev. E 69, 026123 (2004).

[12] A. A. Belavin, A. M. Polyakov, and A. B. Zamolodchikov, J. Stat. Phys. 34, 763 (1984); Nucl. Phys. B 241, 333 (1984); D. Friedan, Z. Qiu, and S. Shenker, Phys. Rev. Lett. 52, 1575 (1984).

[13] J. L. Cardy, in [8], p. 55, and references therein.

[14] T. A. Larsson, J. Phys. A 20, L291 (1987). 
[15] H. Saleur, Nucl. Phys. B 382, 486 (1992).

[16] G. F. Lawler, O. Schramm, and W. Werner, e-print math.PR/ 0108211.

[17] H. J Herrmann and H. E. Stanley, J. Phys. A 21, L829 (1988).

[18] P. Grassberger, J. Phys. A 25, 5475 (1992); Physica A 262, 251 (1999), and references therein.

[19] E. N. Miranda, Physica A 175, 229 (1991).

[20] Y. Deng, H. W. J. Blöte, and B. Nienhuis, Phys. Rev. E 69, 026114 (2003).

[21] J. L. Jacobsen and P. Zinn-Justin, Phys. Rev. E 66, 055102(R) (2002); J. Phys. A 35, 2131 (2002).

[22] M. Porto, A. Bunde, S. Havlin, and H. E. Roman, Phys. Rev. E 56, 1667 (1997).

[23] M. Barthélémy, S. V. Buldyrev, S. Havlin, and H. E. Stanley, Phys. Rev. E 60, R1123 (1999).

[24] G. Paul, S. V. Buldyrev, N. V. Dokholyan, S. Havlin, P. R. King, Y. Lee, and H. E. Stanley, Phys. Rev. E 61, 3435 (2000); G. Paul and H. E. Stanley, ibid. 65, 056126 (2002).

[25] M. Sahimi, Applications of Percolation Theory (Taylor and Francis, London, 1994).

[26] C. D. Lorenz and R. M. Ziff, Phys. Rev. E 57, 230 (1997); G. Paul, R. M. Ziff, and H. E. Stanley, ibid. 64, 026115 (2001).

[27] C. K. Hu, J. A. Chen, N. S. Izmailian, and P. Kleban, Comput.
Phys. Commun. 126, 77 (2000).

[28] H. W. J. Blöte, E. Luijten, and J. R. Heringa, J. Phys. A 28, 6289 (1995); H. W. J. Blöte, J. R. Heringa, A. Hoogland, E. W. Meyer, and T. S. Smit, Phys. Rev. Lett. 76, 2613 (1996); H. W. J. Blöte, L. N. Shchur, and A. L. Talapov, Int. J. Mod. Phys. C 10, 1137 (1999).

[29] M. Hasenbusch, K. Pinn, and S. Vinti, Phys. Rev. B 59, 11471 (1999)

[30] P. Butera and M. Comi, Phys. Rev. B 56, 8212 (1997).

[31] R. Guida and J. Zinn-Justin, J. Phys. A 31, 8103 (1998).

[32] K. Binder and E. Luijten, Phys. Rep. 344, 179 (2001).

[33] M. Campostrini, A. Pelissetto, P. Rossi, and E. Vicari, Phys. Rev. E 65, 066127 (2002).

[34] Y. Deng and H. W. J. Blöte, Phys. Rev. Lett. 88, 190602 (2002); Phys. Rev. E 68, 036125 (2003).

[35] M. D. Rintoul and H. Nakanishi, J. Phys. A 27, 5445 (1994).

[36] Y. Deng and H. W. J. Blöte, Phys. Rev. E 69, 066129 (2004).

[37] M. E. Fisher, Phys. Rev. 176, 257 (1967).

[38] Y. Deng and H. W. J. Blöte (unpublished).

[39] J. R. Heringa and H. W. J. Blöte, Physica A 232, 369 (1995); Phys. Rev. E 57, 4976 (1998).

[40] U. Wolff, Phys. Rev. Lett. 62, 361 (1989). 